\title{
Bimodal Hearing Aid Retention after Unilateral Cochlear Implantation
}

Citation for published version (APA):

Devocht, E. M. J., George, E. L. J., Janssen, A. M. L., \& Stokroos, R. J. (2015). Bimodal Hearing Aid Retention after Unilateral Cochlear Implantation. Audiology and Neurotology, 20(6), 383-393. https://doi.org/10.1159/000439344

Document status and date:

Published: 01/01/2015

DOI:

10.1159/000439344

Document Version:

Publisher's PDF, also known as Version of record

Document license:

Taverne

Please check the document version of this publication:

- A submitted manuscript is the version of the article upon submission and before peer-review. There can be important differences between the submitted version and the official published version of record.

People interested in the research are advised to contact the author for the final version of the publication, or visit the DOI to the publisher's website.

- The final author version and the galley proof are versions of the publication after peer review.

- The final published version features the final layout of the paper including the volume, issue and page numbers.

Link to publication

\footnotetext{
General rights rights.

- You may freely distribute the URL identifying the publication in the public portal. please follow below link for the End User Agreement:

www.umlib.nl/taverne-license

Take down policy

If you believe that this document breaches copyright please contact us at:

repository@maastrichtuniversity.nl

providing details and we will investigate your claim.
}

Copyright and moral rights for the publications made accessible in the public portal are retained by the authors and/or other copyright owners and it is a condition of accessing publications that users recognise and abide by the legal requirements associated with these

- Users may download and print one copy of any publication from the public portal for the purpose of private study or research.

- You may not further distribute the material or use it for any profit-making activity or commercial gain

If the publication is distributed under the terms of Article $25 \mathrm{fa}$ of the Dutch Copyright Act, indicated by the "Taverne" license above, 
Audiology

Neurotology
Audiol Neurotol 2015;20:383-393

DOI: $10.1159 / 000439344$
Received: February 12, 2015

Accepted after revision: August 10, 2015

Published online: October 14, 2015

\title{
Bimodal Hearing Aid Retention after Unilateral Cochlear Implantation
}

\author{
Elke M.J. Devocht ${ }^{a} \quad$ Erwin L.J. George ${ }^{a} \quad$ A. Miranda L. Janssen ${ }^{a, b}$ \\ Robert J. Stokroos ${ }^{\mathrm{a}}$ \\ aDepartment of ENT/Audiology, School for Mental Health and Neuroscience (MHENS), Maastricht University \\ Medical Center, and bepartment of Methodology and Statistics, School for Public Health and Primary Care (CAPHRI), \\ Maastricht University, Maastricht, The Netherlands
}

\section{Key Words}

Bimodal hearing - Patient preference . Cochlear implant . Hearing aid $\cdot$ Residual hearing

\begin{abstract}
The goal of this study was to investigate contralateral hearing aid (HA) use after unilateral cochlear implantation and to identify factors of influence on the occurrence of a unilateral cochlear implant $(\mathrm{Cl})$ recipient becoming a bimodal user. A retrospective cross-sectional chart review was carried out among 77 adult unilateral $\mathrm{Cl}$ recipients 1 year after implantation. A bimodal HA retention rate of $64 \%$ was observed. Associations with demographics, hearing history, residual hearing and speech recognition ability were investigated. Better pure-tone thresholds and unaided speech scores in the non-implanted ear, as well as a smaller difference in speech recognition scores between both ears, were significantly associated with HA retention. A combined model of $\mathrm{HA}$ retention was proposed, and cut-off points were determined to identify those $\mathrm{Cl}$ recipients who were most likely to become bimodal users. These results can provide input to clinical guidelines concerning bimodal $\mathrm{Cl}$ candidacy.
\end{abstract}

(c) 2015 S. Karger AG, Basel
(C) 2015 S. Karger AG, Basel

$1420-3030 / 15 / 0206-0383 \$ 39.50 / 0$

\section{Introduction}

With inclusion criteria broadening over the years [Gifford et al., 2010; Hughes et al., 2014], the number of cochlear implant (CI) candidates keeps increasing. Many still have useful residual hearing in the non-implanted ear and can therefore be fitted with a conventional hearing aid (HA). When electric hearing by means of a CI in 1 ear is supplemented with acoustic hearing by use of a conventional HA in the opposite ear, one speaks of bimodal hearing. This gives several advantages: the use of 2 ears (bilateral), the opportunity to centrally combine the input in both ears (binaural) and access to complementary information. It has been repeatedly demonstrated that bimodal hearing can provide benefit for speech recognition in noise, sound source localization, sound quality and music appreciation [Ching et al., 2007; Olson and Shinn, 2008; El Fata et al., 2009; Schafer et al., 2011].

Many researchers have demonstrated benefit from the HA in bimodal patients. Yet survey studies show that how patients perform in laboratory tests is not always related to how they rate their abilities in everyday situations [Noble et al., 2008]. Thus, more research into stated patient preference regarding bimodal hearing is warranted. For instance, few studies have examined the number and type

\section{KARGER 125}

E-Mail karger@karger.com www.karger.com/aud
Elke M.J. Devocht

Maastricht University Medical Center

Department of ENT/Audiology

PO Box 5800, NL-6202 AZ Maastricht (The Netherlands)

E-Mail elke.devocht@mumc.nl 
of patients who choose to use bimodal hearing in daily life [Cowan and Chin-Lenn, 2004; Fitzpatrick et al., 2009; Yamaguchi and Goffi-Gomez, 2013].

There is uncertainty about the rates of contralateral HA use after unilateral implantation. Most studies report bimodal rates of only 10-25\% [Syms et al., 2002; Tyler et al., 2002; Fitzpatrick et al., 2009; Yamaguchi and GoffiGomez, 2013]. This was countered by a demographic study among 71 adult CI recipients, showing that $75 \%$ tried wearing an HA and 59\% continued using bimodal devices in daily life [Cowan and Chin-Lenn, 2004]. Then again, a recent survey study in CI centres around the world revealed that, within the total population of adult unilateral CI recipients, on average $32 \%$ are bimodal users [Scherf and Arnold, 2014].

Divergent bimodal rates could pertain to particular patient populations or point to differences in clinical practices [Siburt and Holmes, 2015]. There is no consensus on which patients are good candidates for the simultaneous use of a CI and a contralateral HA [Scherf and Arnold, 2014], except the presence of usable residual hearing [Offeciers et al., 2005]. The degree of residual hearing in CI patients however has progressively increased over the years, therefore lower bimodal rates may be related to stricter CI candidacy criteria in the past [Ching, 2005]. A study by Fitzpatrick et al. [2009] showed a significant difference in time since implantation between the unilateral and bimodal patient groups. This study also considered age at implantation, duration of deafness and severity of hearing loss as possible contributing factors. They found a small but significant association between unaided hearing thresholds in the non-implanted ear and the likelihood of using a contralateral HA.

It is known that the prolonged lack of amplification has an effect on functional hearing performance with an HA [Silman et al., 1993]. In that light, it is conceivable that the amount of HA experience before implantation could play a role in subsequently retaining the HA in the contralateral ear. From questionnaire results, it appears that a patient's satisfaction with CI results also influences the bimodal decision process [Fitzpatrick and Leblanc, 2010]. Van Hoesel [2012] showed that the bimodal benefit increased as the HA performance improved relative to that with the CI alone. A study by Yoon et al. [2014] recently suggested that the benefit of a bimodal fitting is facilitated when the performances of both modalities, electric and acoustic, are similar. Therefore, it is plausible that the outcome difference between both ears could influence bimodal HA retention as well.

\section{Current Study}

Studies investigating the added value of an $\mathrm{HA}$ in bimodal patients are numerous. Meanwhile, little research is carried out to assess which unilateral CI recipients are most likely to become bimodal users in the first place. Addressing this question is however very relevant in counselling unilateral CI recipients and providing them with a tailored fitting. The primary goal of the current study was therefore to further investigate the (dis)continuation of contralateral HA use after cochlear implantation and to identify factors contributing to bimodal HA retention.

A retrospective study with a cross-sectional design was carried out within a recent patient population, investigating a large set of factors related to bimodal HA use. Demographic information and HA history were factored in the study. Aside from residual hearing thresholds, it was assumed that also the functional hearing status of the contralateral ear, referring to speech recognition ability, played a role in bimodal HA retention. Furthermore, the outcome with CI and the difference in speech recognition ability between ears were hypothesized to be related to bimodal HA use as well.

The secondary aim of this study was to determine which threshold values best discriminate between HA retention and HA termination. Their determination could be a first step towards clinical guidelines to identify which unilateral CI patients are most likely to retain their HA after receiving a $\mathrm{CI}$ and hence become bimodal users.

\section{Materials and Methods}

\section{Ethics}

This study was conducted in accordance with national legislation, the medical-ethical standards of the local institutional review board and the principles stated in the Declaration of Helsinki. Patients included in the study population declared no objection to the use of clinically obtained data for medical research.

\section{Subjects}

The clinical charts of the adult patients of the CI team SouthEast Netherlands were reviewed. A potential bimodal candidate was defined as a unilateral CI recipient who reported to regularly (more than $50 \%$ of the time) use a conventional HA in the nonimplanted ear prior to receiving the CI. All patients who had received a unilateral CI of the brand Advanced Bionics (Hires $90 \mathrm{~K}$ CI system) in the period 2004-2013 and had come to the CI centre for yearly follow-up were selected $(n=86)$. Within this group, 77 subjects $(89.5 \%)$ were identified as potential bimodal candidates and were therefore included in the study population. In addition to demographic information (sex and age at implantation), details on hearing history were collected: duration of self-reported hear- 
ing loss in the non-implanted ear, HA experience in the non-implanted ear and bilateral HA experience before implantation.

\section{Procedures}

For all patients in the study population, cross-sectional clinical data were reviewed 1 year after cochlear implantation. By then, the outcome situation is deemed to be stable [Luntz et al., 2005, 2007]. Of primary interest was the continuation of HA use in the contralateral ear, referred to as bimodal HA retention. Besides demographic information and details on hearing history, possible associations with the outcome were considered to lie in tone and speech audiometric measures. Results were collected unilaterally with the device in the other ear turned off. When testing the CI ear, the HA was kept in situ whereby the patient's own ear mold acted as a damping plug. For the non-implanted contralateral ear, aided results were never available for those patients who did not continue to use an HA. To ensure consistency, only unaided contralateral pure-tone thresholds and unaided contralateral speech recognition scores were included in the analysis. After all, both unaided and aided measures of speech recognition are known to be related to the same underlying speech recognition ability [Humes, 2002]. All tests were performed in a sound-attenuated room under headphones in the unaided condition, and in free field at ear level at $1 \mathrm{~m}$ distance of a loudspeaker in the aided condition.

\section{Bimodal HA Retention}

Bimodal HA retention was determined to be positive if clinical chart documentation stated that the patient reported to habitually use (more than $50 \%$ of the time) a contralateral HA in combination with the CI 1 year after implantation. Patients with positive HA retention were denoted as the 'bimodal group', while patients who had chosen to discard the HA were denoted as the 'unilateral' group. No strong recommendations were made by the rehabilitation team upon the continuation of contralateral HA use after implantation. HA use was mainly patient-driven and therefore represented stated patient preference regarding bimodal hearing.

During the first weeks after implantation, the primary focus of rehabilitation was the performance with CI. In clinical practice, no systematic bimodal fitting protocol was applied, not even over time, for a simple reason. Although adapting HA and/or CI fitting to optimize bimodal benefit is suggested in the literature [Francart and McDermott, 2013], no generally accepted bimodal fitting method exists. In general, the clinical approach described in this study is characteristic of the current course of bimodal practice in CI centres around the world [Scherf and Arnold, 2014].

\section{Residual Hearing}

Pure-tone audiometric thresholds were clinically collected at octave frequencies from 250 up to $8,000 \mathrm{~Hz}$. If no response was recorded at the maximum output of the audiometer, a value of $5 \mathrm{~dB}$ HL greater than the maximum tested level was noted. The pure-tone average (PTA) was calculated as the mean threshold at $500,1,000$ and 2,000 Hz. For all patients in the study population, the unaided PTA in the non-implanted contralateral ear was determined as a measure of residual hearing ('PTA contra UA'). The threshold at $250 \mathrm{~Hz}$ in the contralateral ear (' $250 \mathrm{~Hz}$ contra UA') was considered as a separate factor. In CI candidates, thresholds at low frequencies commonly show the largest range of residual acoustic hearing. Furthermore, researchers previously demon- strated that mainly low-frequency information $(<500 \mathrm{~Hz})$ contributes to bimodal benefit [Büchner et al., 2009; Mok et al., 2010; Zhang et al., 2010; Illg et al., 2014; Sheffield and Gifford, 2014]. Therefore, residual hearing at low frequencies could relate to bimodal HA retention as well. In the implanted ear, data on aided PTA with CI were gathered ('PTA CI') as a measure of auditory detection outcome with CI.

\section{Speech Recognition}

Speech audiometry was conducted in a quiet setting using a Dutch monosyllabic consonant-nucleus-consonant (CNC) test [Bosman and Smoorenburg, 1995]. The maximum score (\%) was determined over the levels up to $120 \mathrm{~dB}$ SPL for the non-implanted ear in the unaided situation ('CNC contra UA') and over the levels 55, 65 and $75 \mathrm{~dB}$ SPL for the aided condition with $\mathrm{CI}$ (' $\mathrm{CNC}$ CI'). The difference in speech recognition ability between the two ears was also considered to possibly contribute to bimodal HA retention. This difference was defined as the maximum aided score with $\mathrm{CI}$ minus the maximum unaided contralateral score ('CNC $\mathrm{CI}$ - CNC contra UA').

\section{Data Analysis}

Demographic information and clinical test results 1 year after cochlear implantation, for both the bimodal and the unilateral group, were described by means and standard deviations.

Univariable logistic regression analyses were performed to investigate the association between each of the patient characteristics and the status of bimodal HA retention 1 year after cochlear implantation. The univariable odds ratios are presented along with the $95 \%$ statistical confidence intervals and the corresponding $\mathrm{p}$ value. Statistical significance was assumed at $\mathrm{p}<0.05$. The Hosmer and Lemeshow test was performed to inspect the goodness of fit of the logistic model. Since the null hypothesis of this test is a good model fit, a non-significant $\mathrm{p}$ value implies that the model describes the data well.

For each significant contributing factor a receiver-operating characteristic curve (ROC) was studied, and the area under the curve (AUC) was used to assess the ability of the factor to discriminate between positive HA retention and discontinued HA use. An ROC curve plots the true-positive rate (sensitivity) against the false-positive rate ( 1 - specificity) at any given value of the factor under investigation [Metz, 1978; Zweig and Campbell, 1993]. The AUC is equal to the probability that an observer will correctly identify the positive case when presented with a random positive-negative case pair [Hanley and McNeil, 1982]. Hereby an AUC of 0.5 reflects no discrimination above chance, whereas an AUC of 1.0 indicates perfect discrimination. Generally, AUC values of $\geq 0.90$ are considered excellent, $0.80-0.89$ good, $0.70-0.79$ fair and $<0.70$ poor.

For factors with an AUC significantly different from 0.5, the most optimal cut-off point with corresponding specificity and sensitivity was determined using the maximum value of the Youden index [Youden, 1950]. The optimal cut-off point is the discrimination threshold that reflects the best performance of the factor as a classifier of the outcome between positive and negative HA retention, considering the best trade-off between test sensitivity and specificity.

A multivariable logistic regression model was constructed by methods of backward stepwise selection with a drop-out criterion of $\mathrm{p}>0.10$. Those variables that were significantly associated with 
Table 1. Characteristics of the study population and results of univariable logistic regression analysis

\begin{tabular}{|c|c|c|c|c|c|}
\hline \multirow[t]{2}{*}{ Variable } & \multirow{2}{*}{$\begin{array}{l}\text { Bimodal } \\
(\mathrm{n}=49)\end{array}$} & \multirow{2}{*}{$\begin{array}{l}\text { Unilateral } \\
(\mathrm{n}=28)\end{array}$} & \multicolumn{3}{|c|}{ Univariable logistic regression } \\
\hline & & & OR & $95 \% \mathrm{CI}_{\text {stat }}$ & $\mathrm{p}$ \\
\hline Age at implantation, years & $59.9(13.5)$ & $61.2(17.1)$ & 0.99 & $0.96-1.03$ & 0.703 \\
\hline Duration hearing loss contra $(\mathrm{n}=72)$, years & $31.3(16.9)$ & $34.4(14.9)$ & 0.99 & $0.96-1.02$ & 0.434 \\
\hline HA experience contra $(n=60)$, years & $26.9(15.2)$ & $28.2(13.3)$ & 0.99 & $0.96-1.03$ & 0.743 \\
\hline PTA contra UA, dB HL & $92.3(13.0)$ & $102.1(14.0)$ & 0.95 & $0.91-0.98$ & $0.005^{* *}$ \\
\hline $250 \mathrm{~Hz}$ contra UA, dB HL & $76.7(24.4)$ & $88.4(21.9)$ & 0.98 & $0.96-1.00$ & $0.045^{*}$ \\
\hline PTA CI, dB HL & $38.5(9.9)$ & $34.6(9.3)$ & 1.05 & $0.99-1.10$ & 0.099 \\
\hline $\mathrm{CNC}$ contra UA, \% & $38.2(23.6)$ & $16.3(17.9)$ & 1.05 & $1.02-1.07$ & $<0.001^{* *}$ \\
\hline CNC CI, \% & $52.7(25.7)$ & $59.0(28.3)$ & 0.99 & $0.97-1.01$ & 0.318 \\
\hline CNC CI - CNC contra UA, \% & $14.5(33.1)$ & $42.7(26.9)$ & 0.97 & $0.95-0.99$ & $0.001^{* *}$ \\
\hline
\end{tabular}

Patient characteristics at time of implantation and audiological test results 1 year after implantation: mean (with standard deviation) and results of univariable logistic regression analysis for the outcome of HA retention $\left(* \mathrm{p}<0.05,{ }^{* *} \mathrm{p}<0.01\right) . \mathrm{OR}^{*} \mathrm{Odds}$ ratio; $\mathrm{CI}_{\text {stat }}=$ 95\% confidence interval (statistics); contra = non-implanted ear; bil = bilateral; UA = unaided measurement; PTA = pure-tone average at $500,1,000$ and $2,000 \mathrm{~Hz} ; \mathrm{CNC}=$ consonant-nucleus-consonant speech testing in quiet.

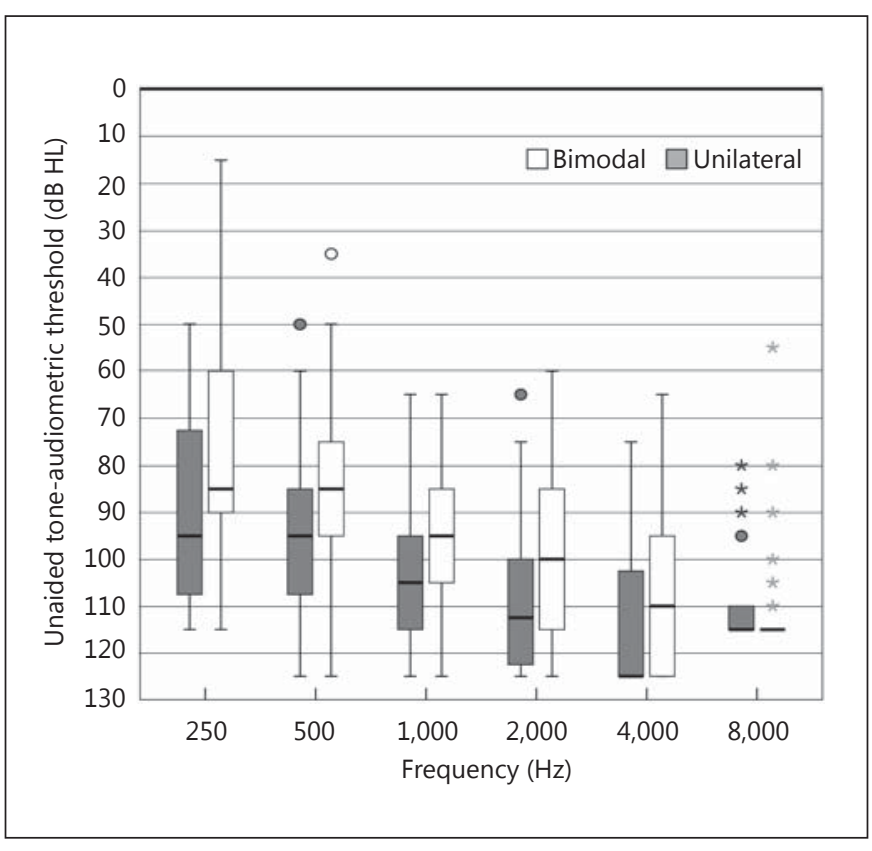

Fig. 1. Residual hearing thresholds in the non-implanted ear for the groups of unilateral and bimodal users 1 year after cochlear implantation. Box plots represent the distribution per frequency (median and interquartile range), with whiskers denoting minimum and maximum values within 1.5 times the interquartile range, circles denoting outliers and asterisks denoting extremes.
HA retention in the univariable analysis were considered candidate factors for the multivariable analysis. Also for the multivariable model, a Hosmer and Lemeshow test and ROC curve analysis were performed, the AUC was assessed, and the optimal cut-off point, as a trade-off between the different remaining factors, was determined.

All statistical analyses were performed using IBM SPSS Statistics version 22.0.0.1.

\section{Results}

\section{Patient Characteristics}

One year after implantation, 49 patients in the study population continued to habitually use an HA in the contralateral ear, while the other 28 patients terminated HA use. This amounts to an HA retention rate of $63.6 \%$.

Demographic information, details on hearing history and audiological test results for the subgroups of bimodal and unilateral users are presented in table 1. For some patients, details on duration of hearing loss and HA experience could not be derived retrospectively from clinical charts. Therefore, the obtained sample size is listed for those variables where it does not equal the total study population.

A visual representation of the hearing loss in the nonimplanted ear for both groups is displayed in figure 1 . The patients who discarded their HA $(\mathrm{n}=28)$ had an average CNC score with the CI of 59\% (SD 28.3\%), while those 

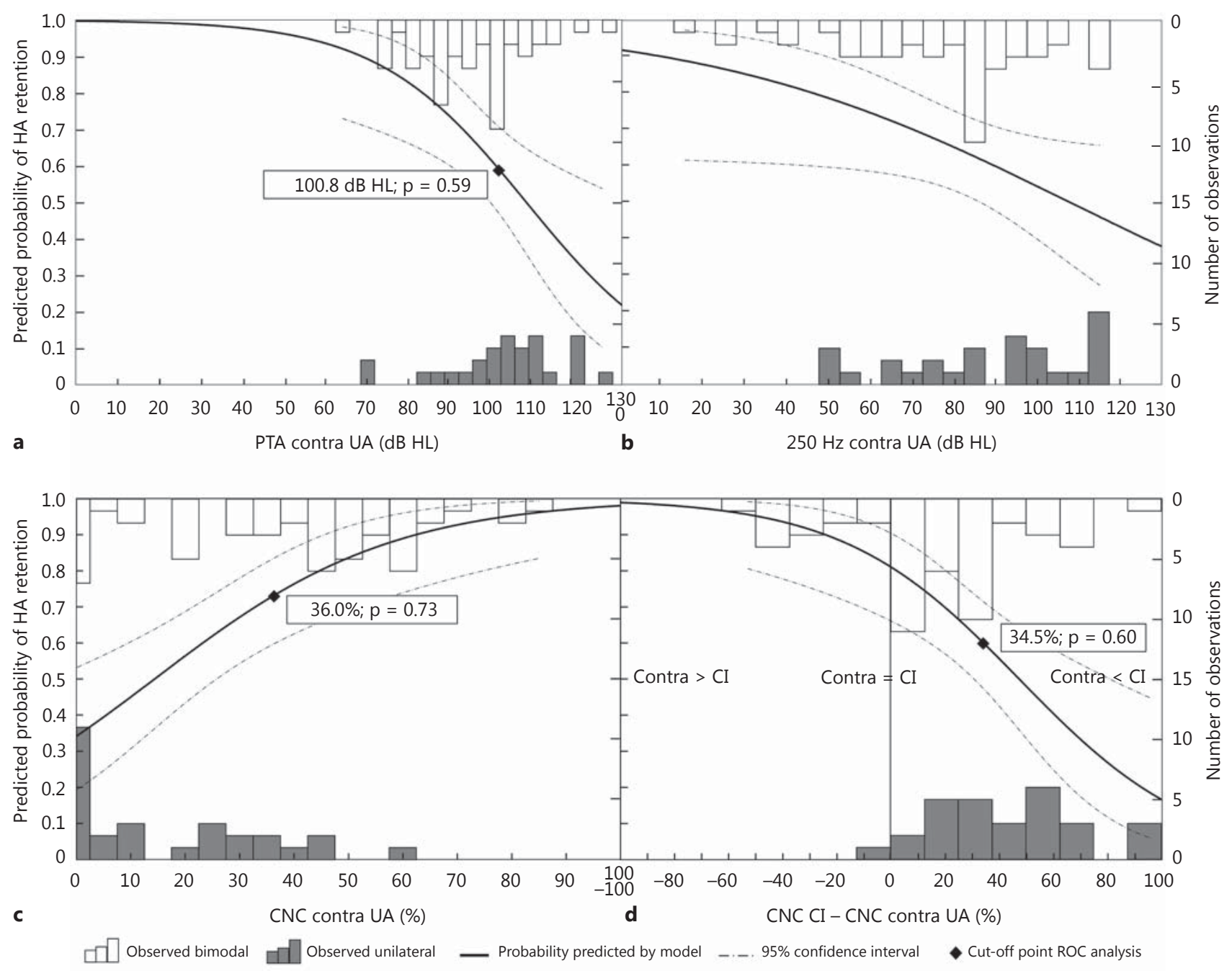

Fig. 2. Probability of bimodal HA retention 1 year after cochlear implantation in relation to the unaided PTA $(500,1,000,2,000 \mathrm{~Hz})$ in the non-implanted contralateral ear (PTA contra UA; a), the unaided pure-tone threshold at $250 \mathrm{~Hz}$ in the non-implanted contralateral ear $(250 \mathrm{~Hz}$ contra UA; b), the maximum unaided CNC score in the non-implanted contralateral ear (CNC contra UA; c) and the difference in maximum speech recognition scores between the cochlear-implanted ear and the unaided contralateral ear (CNC CI - CNC contra UA; d). patients who did retain their HA $(\mathrm{n}=49)$ had average CNC scores of 52.7\% (SD 25.7\%), 41.1\% (SD 23.6\%) and 66.1\% (SD 20.8\%) aided with CI alone, HA alone and CI and HA together, respectively.

\section{Univariable Logistic Regression}

Results of the univariable logistic regression analyses for each factor related to bimodal HA retention are included in table 1 . Age at implantation, duration of con- tralateral hearing loss, experience of HA use in the contralateral ear, bilateral HA experience and PTA and CNC scores with CI did not show a significant relationship with continuation of HA use in the non-implanted ear. Sex had a notable small odds ratio (0.42) in association with HA retention but fell short of significance $(\mathrm{p}=$ 0.079). However, HA retention was shown to be highly significantly related to the general amount of residual hearing in the contralateral ear (PTA contra UA; $\mathrm{p}=$ 
Table 2. Results of ROC analyses with cut-off points

\begin{tabular}{|c|c|c|c|c|c|c|}
\hline Model & AUC & $\mathrm{p}$ & sens. & spec. & pred. prob. & value \\
\hline PTA contra UA & 0.72 & $0.002^{* *}$ & 0.78 & 0.61 & 0.59 & $100.8 \mathrm{~dB} \mathrm{HL}$ \\
\hline $250 \mathrm{~Hz}$ contra UA & 0.63 & 0.053 & & & & \\
\hline CNC contra UA & 0.76 & $<0.001^{* *}$ & 0.59 & 0.86 & 0.73 & $36.00 \%$ \\
\hline CNC contra UA; CNC CI - CNC contra UA & 0.78 & $<0.001^{* *}$ & 0.65 & 0.89 & 0.71 & \\
\hline
\end{tabular}

Results of ROC analysis $\left({ }^{* *} \mathrm{p}<0.01\right)$ for significant univariable factors and the combined multivariable model. For those factors with a significant AUC, cut-off points are determined based on the maximum Youden index. sens. = Sensitivity; spec. = specificity; pred. prob. $=$ predicted probability; contra $=$ non-implanted ear; $\mathrm{UA}=$ unaided measurement; PTA $=$ pure-tone average at $500,1,000$ and $2,000 \mathrm{~Hz} ; \mathrm{CNC}=$ consonant-nucleus-consonant speech testing in quiet.

0.005), the unaided speech scores in the contralateral ear (CNC contra UA; $\mathrm{p}<0.001$ ) and the difference in speech recognition ability between both ears (CNC CI - CNC contra UA; $p=0.001)$. The hearing threshold at $250 \mathrm{~Hz}$ ( $250 \mathrm{~Hz}$ contra UA) also showed an association with HA retention, although in comparison to the other factors it was found to be only just significant ( $p=0.045)$. Hosmer and Lemeshow tests were not significant ( $p>0.05)$, indicating a good fit for each of the univariable models.

Since HA use was defined as positive if an HA is retained after implantation, a significant odds ratio $>1$ means that HA retention was associated with higher values of the variable under investigation. On the other hand, an odds ratio $<1$ stands for an association between HA retention and lower values of the tested variable. As shown in table 1, resulting odds ratios revealed that the probability of bimodal HA retention increased with lower (i.e. better) residual hearing thresholds (averaged and at $250 \mathrm{~Hz}$ ) and higher speech recognition scores in the contralateral ear. HA continuation was also related to a smaller difference in speech scores, referring to a functional outcome whereby the $\mathrm{CI}$ is less dominant over the HA ear. Figure $2 \mathrm{a}-\mathrm{d}$ illustrates the results for each of the four factors found to be significantly related to bimodal HA retention. Histograms represent the observed distribution of the factor under investigation for the bimodal group (open bars at the top) and for the unilateral group (shaded bars at the bottom). The fitted line, accompanied by its $95 \%$ confidence range, demonstrates the probability of bimodal HA retention predicted by the logistic model of the respective factors.

\section{Univariable ROC Analysis}

Table 2 summarizes the results of the univariable ROC analysis. With significant AUC values of $0.72-0.76$, the factors PTA contra UA, CNC contra UA and CNC $\mathrm{CI}$ - CNC contra UA exhibited fair accuracy in discriminating between positive and negative HA retention. For the factor $250 \mathrm{~Hz}$ contra UA however, discrimination accuracy was not significantly better than chance in defining HA retention $(\mathrm{p}=0.053)$. This is related to the fact that the contralateral threshold at $250 \mathrm{~Hz}$ was only just significant in the earlier univariable logistic regression analysis.

For each of the other three significant factors, a cut-off point was determined, as displayed in figure $2 a, c$ and $d$. Evaluation of the cut-off points demonstrated that a PTA in the contralateral ear of less than $100.8 \mathrm{~dB} \mathrm{HL}$, an unaided speech recognition score in the non-implanted ear of more than $36 \%$ and a speech recognition difference of less than $34.5 \%$ in favour of the CI were associated with continued HA use after implantation.

\section{Multivariable Logistic Regression}

To investigate the relative combined contribution of the variables PTA contra UA, $250 \mathrm{~Hz}$ contra UA, CNC contra UA and CNC CI - CNC contra UA to bimodal HA retention, backward stepwise selection was applied to construct a multivariable logistic regression model:

$$
\begin{aligned}
& \mathrm{P}(\text { HA retention })=1 /(1+\mathrm{EXP}-[0.03(\mathrm{CNC} \text { contra UA })- \\
& 0.02(\mathrm{CNC} \mathrm{CI}-\mathrm{CNC} \text { contra UA })+0.21]) .
\end{aligned}
$$

Hereby EXP indicates the natural exponential function, and $\mathrm{P}$ stands for the predicted probability of HA retention from the regression model. This model (equation 
Fig. 3. Probability of bimodal HA retention 1 year after cochlear implantation in relation to the maximum speech recognition score in the cochlear implanted ear (CNC $\mathrm{CI})$ and the unaided contralateral ear (CNC contra UA).

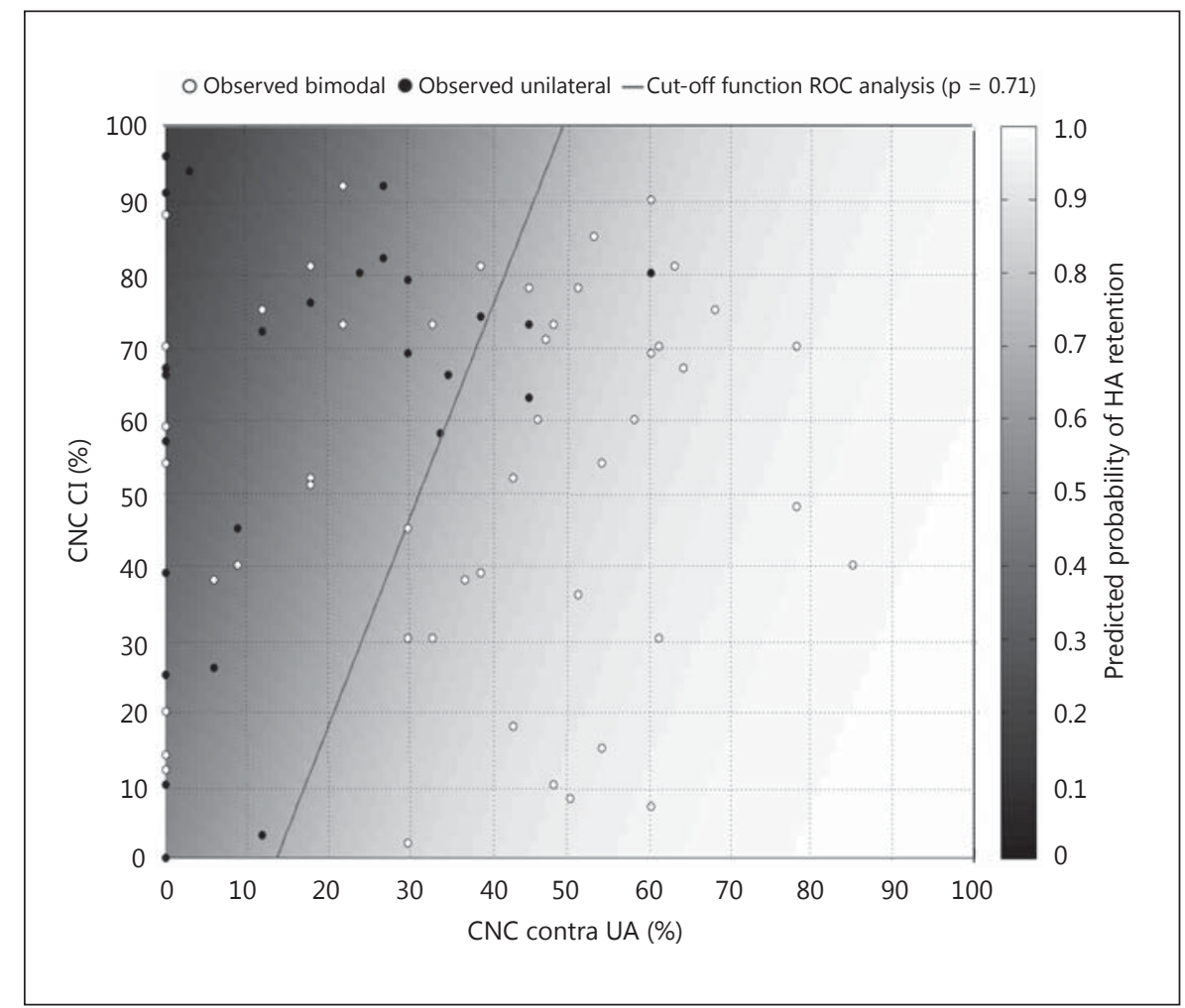

1) included both speech recognition in the contralateral ear (odds ratio 1.03, confidence interval 1.00-1.06, $\mathrm{p}=$ 0.025 ) and the difference between both ears (odds ratio 0.98 , confidence interval $0.96-1.00, p=0.096$ ) as significantly contributing variables. However, the factors representing the mean unaided pure-tone audiometric threshold and the threshold at $250 \mathrm{~Hz}$ did not improve the predictive power. They were therefore not selected for inclusion in the combined regression model ( $p=0.94$ and $\mathrm{p}=0.82$, respectively). A Hosmer and Lemeshow test on the final model did not yield significance ( $p>0.05)$, indicating a good fitted model. The fitted multivariable logistic model is illustrated in figure 3 . The greyscale represents the modelled probability of contralateral HA retention, with brightness indicating a higher predicted probability of bimodal HA continuation. The circles, which display the original data of the study population, are filled for subjects who discontinued HA use (unilateral group) and open for subjects who continued HA use (bimodal group).

\section{Multivariable ROC Analysis}

The result of ROC curve analysis for the multivariable model is shown in table 2 . The model discriminated with significant and fairly good accuracy (AUC 0.78) between positive and negative HA retention. The cut-off point function for $\mathrm{HA}$ retention based on the combination of outcome measures $\mathrm{CNC}$ contra $\mathrm{UA}$ and $\mathrm{CNC} \mathrm{CI}$ is associated with a predicted probability of 0.71 and is illustrated by the fitted line in figure 3 . Within the range limits of the CNC outcome measures (0-100\%), this cut-off point function demonstrated that a maximum $\mathrm{CNC}$ word score $<13.8 \%$ in the unaided contralateral ear was associated with discontinued HA use after implantation. In contrast, a maximum CNC score $>49 \%$ in the unaided HA ear was associated with continued HA use. For an unaided CNC score in the non-implanted ear between 13.8 and $49 \%$, the CNC outcome with CI played a complementary role in the association with bimodal HA retention.

\section{Discussion}

\section{Bimodal HA Retention}

The aim of this study was to investigate the continuation of contralateral HA use after unilateral cochlear implantation. For patients who wore a contralateral HA preoperatively, an HA continuation rate of $64 \%$ after 1 year of CI experience was observed. This bimodal rate is rath- 
er higher than the range of bimodal use (10 up to 59\%) retrieved from previous demographic studies [Syms et al., 2002; Tyler et al., 2002; Cowan and Chin-Lenn, 2004]. Previous studies never noted whether an HA had been used prior to implantation and therefore most likely looked into the total unilateral population. If the total unilateral population is considered in this study, still a rather high bimodal usage rate of 57\% (49 out of 86 subjects) was found. Unlike some of those previous studies, the present study placed no predefined restrictions on residual hearing for bimodal eligibility, yet a higher rate of HA retention was found. The higher rate is most likely an effect of inclusion criteria becoming less strict over the years. All previous studies cover patients implanted before 2004, while this study population contained more recent CI recipients (2004-2013). Its composition accounts for the difference in mean contralateral residual hearing thresholds (PTA) between this recent study population (95.9 dB HL) and that of a previous study (108.1 dB HL) by Fitzpatrick et al. [2009].

\section{Contributing Factors}

Residual Hearing

The degree of residual contralateral hearing turned out to be significantly associated with HA retention. This is consistent with previous research [Yamaguchi and GoffiGomez, 2013], just as the finding that average contralateral residual hearing (PTA) was a contributing factor, while the threshold specified at low frequencies did not seem to add any additional information [Fitzpatrick et al., 2009]. Some earlier studies showed that low-frequency information contributes to bimodal benefit for speech recognition in noise, prosody and music perception since it is not well transmitted by the CI [Büchner et al., 2009; Mok et al., 2010; Illg et al., 2014]. However, such complex auditory tasks were not addressed in the current study, which looked into the reasons for patients to become bimodal or unilateral users in the first place. Here results suggest usable residual hearing over a wide frequency range as reason for patients to retain their HA. This result can be related to other findings from the bimodal literature that state that a contralateral HA should be fitted to provide acoustic information over the whole range of frequencies with usable residual hearing, and not only at low frequencies [Neuman and Svirsky, 2013; Sheffield and Gifford, 2014].

\section{Speech Recognition}

Speech recognition ability in the non-implanted ear also appeared to be related to HA retention after implantation. In fact, the multivariable model demonstrated that mainly speech recognition, assessed by the maximum CNC word recognition score in the non-implanted ear and the difference between both ears, was related to bimodal HA use. Tone audiometric hearing level did not add any additional information to the combined model. Although auditory detection is a prerequisite for functional hearing, these findings point to speech recognition as a more important factor contributing to bimodal HA retention in daily life.

Besides threshold information, it has been suggested that measures of suprathreshold information are also important for speech recognition [Stephens, 1976; Plomp, 1978; Glasberg and Moore, 1989; George et al., 2006]. Hearing-impaired subjects with comparable hearing sensitivity (i.e. pure-tone thresholds) would not necessarily achieve the same level of speech recognition [Skinner, 1982]. The results of the current study highlight the importance of suprathreshold factors for the bimodal research field. Suprathreshold hearing incorporates functions such as frequency resolution, temporal resolution, temporal fine structure coding and loudness perception, but also linguistic skills and cognition [Kramer et al., 2009; Bernstein and Mehraei, 2013; Grant and Walden, 2013]. As Zhang et al. [2013] recently suggested in a study on bimodal benefit, acoustic spectral modulation detection thresholds, as a measure of spectral resolution, may be stronger predictors of the degree of benefit than basic audiometric measurements.

Although in this study speech recognition proved to be the main factor associated with bimodal HA retention, it could not explain continued bimodal use for all patients. Figures $2 \mathrm{c}$ and 3 show that some subjects did continue to wear a contralateral HA, despite having little or no measurable speech recognition ability in the nonimplanted ear. For those patients, other qualities of the acoustic ear may be important for retaining the HA, depending on the demands of their environment. For example, Kong et al. [2005] described 5 patients who, even though no speech recognition could be achieved with the acoustic ear alone, gained benefit from bimodal stimulation when listening to speech in background noise. This benefit was attributed to the ability of the acoustic ear to analyse the temporal fine structure of the acoustic scene.

\section{Difference between Ears}

It has previously been suggested [Ching et al., 2004; Noble et al., 2008], and demonstrated in a survey study [Fitzpatrick and Leblanc, 2010], that CI outcome plays an important role in the bimodal decision-making pro- 
cess. When results with the CI are unsatisfying, a contralateral HA may provide adequate means to improve performance in daily listening situations. On the other hand, when CI results are already satisfying, an HA might be experienced as interfering with the outperformance of the CI.

Data from the current study suggest that it is not the CI outcome by itself but the difference between functional speech performance of both ears that significantly affects HA retention. It was seen that a between-ear difference score less in favour of the CI was related to HA continuation after implantation. If the HA outperforms the $\mathrm{CI}$, in case of a disappointing CI outcome, it seems evident that a patient will decide to continue HA use. However, the distribution of difference scores among bimodal users (fig. 2d) reveals that most bimodal users had a comparable speech recognition ability in both ears (difference between 0 and 30\%). Apparently, functional across-ear comparability is connected to bimodal use. This observation is related to a recent study by Yoon et al. [2015] on the benefit provided by a bimodal fitting. They demonstrated a significant correlation between bimodal benefit and the difference in performance between ears for speech recognition, with a smaller difference leading to greater bimodal benefit.

Finally, the importance of the difference between ears found in this study suggests that emerging patient preference takes the contralateral score as a reference point for performance. The postoperative CI outcome may then be weighed against that score when making the decision whether to opt for bimodal hearing.

\section{Gender}

Sex approached but did not reach significance as a factor in the relationship with HA retention in the current study. Despite limited power, a trend towards male subjects having a lower rate of bimodal HA retention could be observed (table 1). The underlying reasons for the found trend are unclear. A gender difference might be intermediated by the degree of hearing loss or related to attitude factors such as appearance. Even in the general field of HAs, there has been very little research to date that examines the differences between men and women in the use of HAs [McCormack and Fortnum, 2013]. In a study by Staehelin et al. [2011], women also reported a higher prevalence of daily and regular use of HAs.

\section{Design Limitations}

An even better representation of functional hearing would have been based on the aided speech reception scores with an HA. Indeed, it is reasonable to assume that HA fitting could also have played a role in the observed bimodal HA retention rate since insufficient gain could restrain the perceived bimodal benefit [Yehudai et al., 2013]. However, the current retrospective study design of a cross-sectional review 1 year after implantation implied that postoperative aided data in the contralateral ear were not available for unilateral patients who discarded their HA. Furthermore, also pure-tone thresholds below 250 $\mathrm{Hz}$ were unavailable, since these are not routinely measured in clinical practice. A limitation of the current study therefore lies in its retrospective cross-sectional design in a clinical population. Moreover, since the outcome situation and the possible contributing factors were all collected at the same point in time (1 year after implantation), a predictive model in the strict sense of the word was not possible. Nevertheless, this design was chosen because it enabled exclusion of progressive hearing loss as a confounding factor and inclusion of the outcome with a CI. Recent research has demonstrated stability of contralateral residual hearing up to $2,000 \mathrm{~Hz}$ over more than 6 years after implantation [Yehudai et al., 2012]. This means that hearing status 1 year after implantation can be considered a good estimate of pre-operative hearing abilities.

\section{Evaluation of Study Aims}

The primary aim of the current study was to investigate demographic and auditory factors related to bimodal HA retention. However, many non-auditory factors are known to impact HA satisfaction, such as aesthetics, stigma, habitude, cost and comfort [Wong, 2003]. These latter factors contribute to the stated patient preference and may account for some of the unaccounted predictive power of the current model. Incorporation of more factors would require a larger cohort and self-reported experiences with bimodal fitting. To this end, and to investigate follow-up use in the longer term, further research is warranted.

The secondary aim was to determine discrimination values to identify which unilateral CI patients are most likely to become bimodal users. Results are based on standard data from CI clinical practice. Therefore, these statistical outcomes can provide input for clinical guidelines on bimodal CI candidacy. Besides underpinning decision-making on which ear to implant, the outcomes can help distinguish between potential bimodal candidates and candidates for bilateral implantation.

For residual hearing (PTA), a cut-off point of $100 \mathrm{~dB}$ HL was obtained. This lies within the range of criteria (from $<90$ to $110 \mathrm{~dB} \mathrm{HL}$ ) applied in bimodal research to define usable residual hearing [Fitzpatrick et al., 2009; 
Park et al., 2012; Neuman and Svirsky, 2013]. For speech recognition ability in the contralateral ear, a cut-off point of $36 \%$ was found, or a point somewhere between 14 and $49 \%$ when looking at the model combined with the outcome of the CI. These results align with previous findings: subjects with pre-operative contralateral HA word scores of roughly $\geq 20 \%$ tend to have greater potential to derive benefit from bimodal stimulation than subjects scoring $<20 \%$ [Morera et al., 2005].

It may be inferred from these cut-off points that, with a CNC score of $>49 \%$ bimodal stimulation stands a good chance, that a score of $<14 \%$ warrants considering bilateral implantation, and that a score somewhere in between calls for considering the degree of achieved bimodal benefit before deciding upon bilateral implantation. It was also found that patients with more balanced speech recognition abilities (less than 35\% difference between the $\mathrm{CI}$ and non-implanted ear) have more chances of becoming a bimodal user. This result is in line with the findings of Yoon et al. [2015], who suggested that a speech performance difference of more than $30 \%$ would potentially cause bimodal interference.

Determining cut-off points in the univariable and multivariable ROC analysis is only the first step towards formulating clinical guidelines. These cut-off points have to be tested for validity in other patient populations and in a prospective manner before they could be relied upon as rules in everyday clinical practice.

\section{Conclusions}

This study investigated contralateral HA use after unilateral implantation. A retrospective cross-sectional chart review demonstrated a $64 \%$ rate of bimodal HA retention 1 year after implantation. Residual hearing and mainly speech recognition ability were identified as factors related to the occurrence of a unilateral CI recipient becoming a bimodal user 1 year after implantation. Patients who had more residual speech recognition ability in the nonimplanted ear and featured a less dominant outcome of the $\mathrm{CI}$ over the HA ear were more likely to continue using a contralateral HA 1 year after receiving a unilateral CI. Although the criteria for bimodal candidacy are still unresolved, this study proposed discrimination values to identify which unilateral CI patients are most likely to turn into bimodal users.

\section{Disclosure Statement}

We acknowledge a research grant from Advanced Bionics Inc., which financially supported the work of the first author (E.D.) in this investigator-initiated study. The third author (M.J.) provided statistical support, made possible by a grant from the Dutch Heinsius-Houbolt Foundation. The authors declare no other conflicts of interest.

\section{References}

Bernstein J, Mehraei G: Spectrotemporal modulation sensitivity as a predictor of speech intelligibility for hearing-impaired listeners. J Am Acad Audiol 2013;24:293-306.

Bosman A, Smoorenburg G: Intelligibility of Dutch CVC syllables and sentences for listeners with normal hearing and with three types of hearing impairment. Audiology 1995;34: 260-284.

Büchner A, Schüssler M, Battmer RD, Stöver T, Lesinski-Schiedat A, Lenarz T: Impact of lowfrequency hearing. Audiol Neurootol 2009; 14:8-13.

Ching T: The evidence calls for making binauralbimodal fittings routine. Hear J 2005;58:3241.

Ching TY, Incerti P, Hill M: Binaural benefits for adults who use hearing aids and cochlear implants in opposite ears. Ear Hear 2004;25:921.

Ching TY, van Wanrooy E, Dillon H: Binauralbimodal fitting or bilateral implantation for managing severe to profound deafness: a review. Trends Amplif 2007;11:161-192.
Cowan R, Chin-Lenn J: Pattern and prevalence of hearing aid use post-implantation in adult cochlear implant users. Aust NZ J Audiol Suppl 2004;May:48.

El Fata F, James CJ, Laborde M-L, Fraysse B: How much residual hearing is 'useful' for music perception with cochlear implants? Audiol Neurootol 2009;14(suppl 1):14-21.

Fitzpatrick EM, Leblanc S: Exploring the factors influencing discontinued hearing aid use in patients with unilateral cochlear implants. Trends Amplif 2010;14:199-210.

Fitzpatrick EM, Seguin C, Schramm D, Chenier J, Armstrong S: Users' experience of a cochlear implant combined with a hearing aid. Int J Audiol 2009;48:172-182.

Francart T, McDermott HJ: Psychophysics, fitting, and signal processing for combined hearing aid and cochlear implant stimulation. Ear Hear 2013;34:685-700.

George E, Festen J, Houtgast T: Factors affecting masking release for speech in modulated noise for normal-hearing and hearing-impaired listeners. J Acoust Soc Am 2006;120:2295-2311.
Gifford RH, Dorman MF, Shallop JK, Sydlowski SA: Evidence for the expansion of adult cochlear implant candidacy. Ear Hear 2010;31: 186-194.

Glasberg B, Moore B: Psychoacoustic abilities of subjects with unilateral and bilateral cochlear hearing impairments and their relationship to the ability to understand speech. Scand Audiol 1989;32:1-25.

Grant K, Walden T: Understanding excessive SNR loss in hearing-impaired listeners. J Am Acad Audiol 2013;24:329-336.

Hanley J, McNeil B: The meaning and use of the area under a receiver operating characteristic (ROC) curve. Radiology 1982;143:29-36.

Hughes MLM, Neff DDL, Simmons JL, Moeller MP: Performance outcomes for borderline cochlear implant recipients with substantial preoperative residual hearing. Otol Neurotol 2014;35:1373-1384.

Humes LE: Factors underlying the speech-recognition performance of elderly hearing-aid wearers. J Acoust Soc Am 2002;112:11121132. 
Illg A, Bojanowicz M, Lesinski-Schiedat A, Lenarz T, Büchner A: Evaluation of the bimodal benefit in a large cohort of cochlear implant subjects using a contralateral hearing aid. Otol Neurotol 2014;35:e240-e244.

Kong Y, Stickney G, Zeng F: Speech and melody recognition in binaurally combined acoustic and electric hearing. J Acoust Soc Am 2005; 117:1351-1361.

Kramer SE, Zekveld A, Houtgast T: Measuring cognitive factors in speech comprehension: the value of using the Text Reception Threshold test as a visual equivalent of the SRT test. Scand J Psychol 2009;50:507-515.

Luntz M, Shpak T, Weiss H: Binaural-bimodal hearing: concomitant use of a unilateral cochlear implant and a contralateral hearing aid. Acta Otolaryngol 2005;125:863-869.

Luntz M, Yehudai N, Shpak T: Hearing progress and fluctuations in bimodal-binaural hearing users (unilateral cochlear implants and contralateral hearing aid). Acta Otolaryngol 2007; 127:1045-1050.

McCormack A, Fortnum H: Why do people fitted with hearing aids not wear them? Int J Audiol 2013;52:360-8.

Metz C: Basic principles of ROC analysis. Semin Nucl Med 1978;8:283-298.

Mok M, Galvin KL, Dowell RC, McKay CM: Speech perception benefit for children with a cochlear implant and a hearing aid in opposite ears and children with bilateral cochlear implants. Audiol Neurootol 2010;15:44-56.

Morera C, Manrique M, Ramos A, Garcia-Ibanez L, Cavalle L, Huarte A, et al: Advantages of binaural hearing provided through bimodal stimulation via a cochlear implant and a conventional hearing aid: a 6-month comparative study. Acta Otolaryngol 2005;125:596-606.

Neuman AC, Svirsky M: Effect of hearing aid bandwidth on speech recognition performance of listeners using a cochlear implant and contralateral hearing aid (bimodal hearing). Ear Hear 2013;34:553-561.

Noble W, Tyler R, Dunn C, Bhullar N: Unilateral and bilateral cochlear implants and the implant-plus-hearing-aid profile: comparing self-assessed and measured abilities. Int J Audiol 2008;47:505-514.
Offeciers E, Morera C, Müller J, Huarte A, Shallop J, Cavallé L: International consensus on bilateral cochlear implants and bimodal stimulation. Acta Otolaryngol 2005;125:918-919.

Olson AD, Shinn JB: A systematic review to determine the effectiveness of using amplification in conjunction with cochlear implantation. J Am Acad Audiol 2008;19:657-671.

Park LR, Teagle HFB, Buss E, Roush PA, Buchman CA: Effects of frequency compression hearing aids for unilaterally implanted children with acoustically amplified residual hearing in the nonimplanted ear. Ear Hear 2012;33:e1-e12.

Plomp R: Auditory handicap of hearing impairment and the limited benefit of hearing aids. J Acoust Soc Am 1978;63:533-549.

Schafer EC, Amlani AM, Paiva D, Nozari L, Verret S: A meta-analysis to compare speech recognition in noise with bilateral cochlear implants and bimodal stimulation. Int J Audiol 2011;50:871-880.

Scherf FW, Arnold LP: Exploring the clinical approach to the bimodal fitting of hearing aids and cochlear implants: results of an international survey. Acta Otolaryngol 2014;134: 1151-1157.

Sheffield SW, Gifford RH: The benefits of bimodal hearing: effect of frequency region and acoustic bandwidth. Audiol Neurootol 2014; 19:151-163.

Siburt HW, Holmes AE: Bimodal fitting: a survey of current clinical practice. Am J Audiol 2015, Epub ahead of print.

Silman S, Silverman CA, Emmer MB, Gelfand SA: Effects of prolonged lack of amplification on speech-recognition performance: preliminary findings. J Rehabil Res Dev 1993;30:326332.

Skinner M: Amplification bandwidth and speech intelligibility for two listeners with sensorineural hearing loss. Audiol J Audit Commun 1982;21:251-268.

Staehelin K, Bertoli S, Probst R, Schindler C, Dratva J, Stutz EZ: Gender and hearing aids: patterns of use and determinants of nonregular use. Ear Hear 2011;32:e26-e37.

Stephens S: The input for a damaged cochlea - a brief review. Br J Audiol 1976;10:97-101.
Syms C, Wickesberg J, Kubo T, Takahashi Y, Iwaki T: Concurrent use of cochlear implants and hearing aids; in Kubo T, Takahashi Y, Iwaki T (eds): Cochlear Implants - An Update. The Hague, Kugler Publications, 2002, pp 535539.

Tyler RS, Parkinson AJ, Wilson BS, Witt S, Preece JP, Noble W: Patients utilizing a hearing aid and a cochlear implant: speech perception and localization. Ear Hear 2002;23:98-105.

Van Hoesel RJM: Contrasting benefits from contralateral implants and hearing aids in cochlear implant users. Hear Res 2012;288:100-113.

Wong LLN: Hearing aid satisfaction: what does research from the past 20 years say? Trends Amplif 2003;7:117-161.

Yamaguchi CT, Goffi-Gomez MVS: Prevalence of contralateral hearing aid use in adults with cochlear implants. Int Arch Otorhinolaryngol 2013;17:370-374.

Yehudai N, Shpak T, Most T, Luntz M: Functional status of hearing aids in bilateral-bimodal users. Otol Neurotol 2013;34:675-681.

Yehudai N, Shpak T, Most T, Luntz M: Natural history of contralateral residual hearing in unilateral cochlear implant users - long-term findings. Acta Otolaryngol 2012;132:10731076.

Yoon Y-S, Shin Y-R, Gho J-S, Fu Q-J: Bimodal benefit depends on the performance difference between a cochlear implant and a hearing aid. Cochlear Implants Int 2015;16:159167.

Youden W: Index for rating diagnostic tests. Cancer 1950;3:32-35.

Zhang T, Dorman MF, Spahr AJ: Information from the voice fundamental frequency (F0) region accounts for the majority of the benefit when acoustic stimulation is added to electric stimulation. Ear Hear 2010;31:63-69.

Zhang T, Spahr AJ, Dorman MF, Saoji A: Relationship between auditory function of nonimplanted ears and bimodal benefit. Ear Hear 2013;34:133-141.

Zweig M, Campbell G: Receiver-operating characteristic (ROC) plots: a fundamental evaluation tool in clinical medicine. Clin Chem 1993;39:561-577. 